\section{Paralogie et redondance : maintenir l'intégrité du génome au cours de la recombinaison V(D)J}

\author{
Chloé Lescale, Hélène Lenden Hasse, Ludovic Deriano
}

Département d'immunologie et département de génomes et génétique, Institut Pasteur, 25, rue du Docteur Roux, 75015 Paris, France. ludovic.deriano@pasteur.fr

par ATM, de nombreuses protéines et de la formation de foyers de réparation. Chez l'homme, comme chez la souris, une déficience de la protéine ATM conduit au syndrome ataxia telangiectasia ${ }^{1}$ qui se traduit, entre autre, par un affaiblissement du système immunitaire et une prédisposition aux cancers. Malgré cela, les cellules lymphocytaires déficientes pour ATM sont capables de terminer leur développement et de produire des récepteurs pour les antigènes fonctionnels.

Une deuxième étape majeure de la recombinaison $V(D)$ J est la réparation des DSB par la voie de réparation non-homologue ou NHEJ (nonhomologous end-joining) [4,

$(\rightarrow)$ Voir la Nouvelle de P. Revy et JP. De Villartay, $m / s n^{\circ}$ 6-7, juin-

Clivage, signalisation et réparation les principales étapes de la recombinaison $\mathrm{V}(\mathrm{D}) \mathrm{J}$ et ses acteurs La recombinaison $V(D)$ J a lieu pendant la phase Gl du cycle cellulaire. Elle est initiée par la formation de DSB induites par la nucléase RAGl/2 au niveau des séquences signal de recombinaison (RSS) qui flanquent les segments codants $V, D$ et J [2]. Les DSB sont alors rapidement reconnues par la voie de réponse aux dommages de I'ADN (DDR, DNA damage response) qui dépend en grande partie de la protéine kinase ATM (ataxiatelangiectasia mutated) [3]. Au-delà de l'activation du point de contrôle Gl/S du cycle cellulaire dépendant de p53, ATM contribue également à la réparation des DSB, notamment en participant à la stabilisation des extrémités d'ADN au sein d'un complexe post-clivage (PCC, post-cleavage complex). Cette fonction semble dépendre de la phosphorylation,
5] $(\rightarrow)$. Au cours de la NHEJ, I'hétérodimère $\mathrm{Ku} 70 / 80(\mathrm{Ku})$ se lie aux extrémités d'ADN et sert de plateforme pour le recrutement de multiples protéines dont la protéine kinase ADN-PKcs (DNAdependent protein kinase, catalytic subunit) et l'endonucléase Artémis, qui permettent de modifier les extrémités d'ADN codantes avant ligature. Ku recrute également le complexe protéique formé par l'ADN ligase IV (LIG4), XRCC4 (X-ray repair cross-complementing protein 4) et XLF (XRCC4-like factor; aussi appelé Cernunnos [5]), qui permet la ligature de ces extrémités (Figure 1). Les modèles de souris, ainsi que les patients présentant une déficience

\footnotetext{
${ }^{1}$ L'ataxie télangiectasie (ou syndrome de Louis-Bar) est une maladie génétique autosomique récessive avec des symptômes neurologiques et immunitaires.
} 


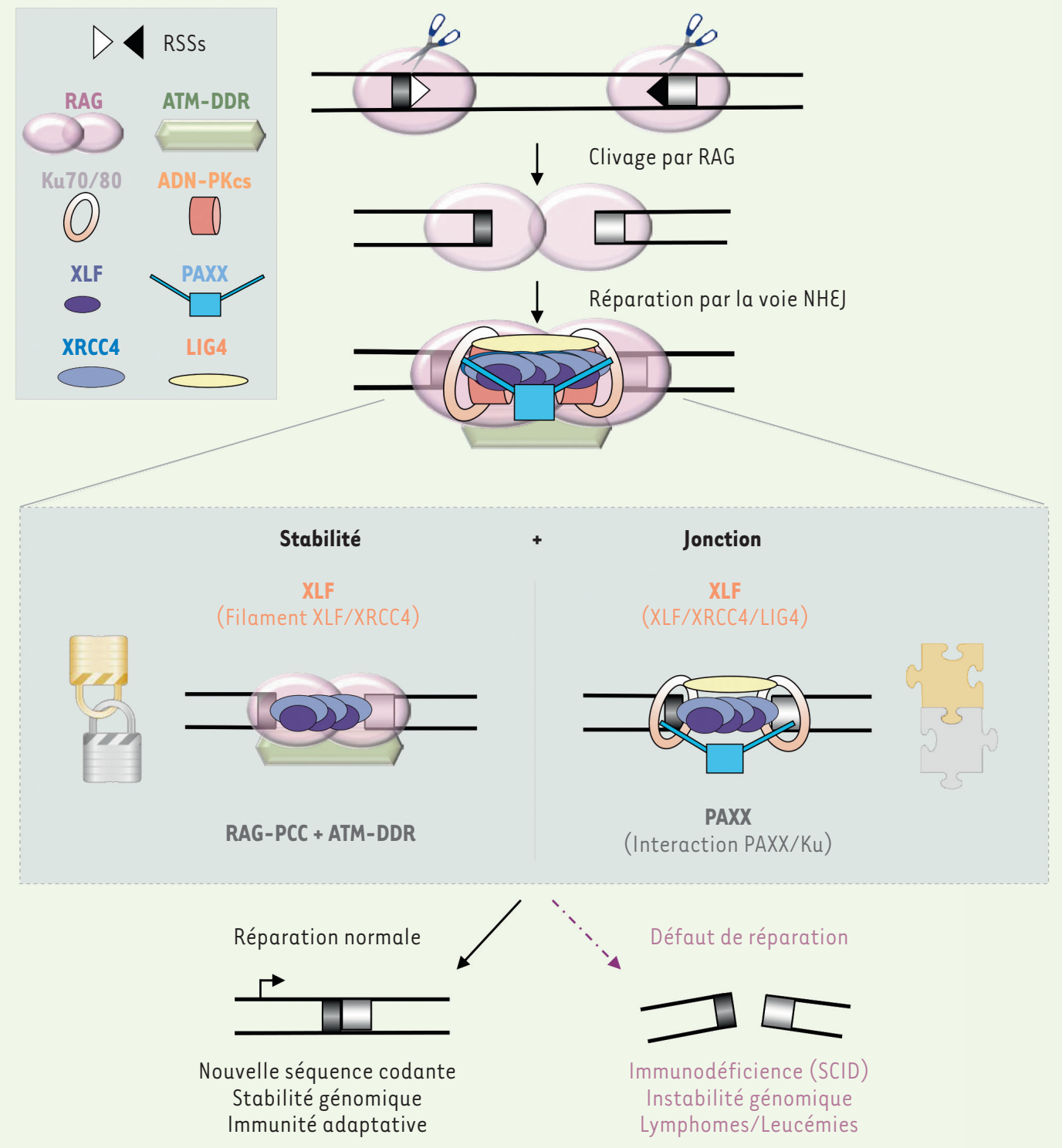

Figure 1. Redondance fonctionnelle entre différents acteurs de la recombinaison $V(D) J$ : une double sécurité pour prévenir un défaut du système immunitaire et une instabilité génomique. Au cours de la recombinaison V(D)J, les protéines RAG1 et RAG2 (RAG, recombination-activating gene) restent associées aux cassures de l'ADN dans un complexe post-clivage (PCC, post-cleavage complex) RAG-PCC. XLF (XRCC4-like factor), RAG et la voie de réponse aux dommages de I'ADN dépendante d'ATM (ataxia-telangiectasia mutated) ATM-DDR (DNA damage response) favorisent la jonction des cassures de l'ADN en les stabilisant au sein du PCC. PAXX et XLF participent à la ligature des extrémités par la voie NHEJ (non-homologous end-joining). Une déficience combinée de (1) RAG2 et XLF, (2) ATM et XLF ou (3) PAXX et XLF, conduit à une immunodéficience sévère et à une instabilité génomique prédisposant au développement de tumeurs lymphoïdes. RSS : recombination signal sequence ; DNA-PKcs : DNA-dependent protein kinase catalytic subunit; RS-SCID : radiation sensitive - severe combined immunodeficiency ; XRCC4 : X-ray repair cross-complementing protein 4 ; LIG4 : ADN ligase IV ; Ku : hétéro-dimère Ku70/80.

héréditaire de la voie NHEJ, souffrent d'une grande variété de dysfonctionnements, notamment : (a) une sensibilité accrue aux agents génotoxiques, (b) une immunodéficience combinée sévère, communément appelée SCID (severe combined immunodeficiency), due à un défaut de réparation des DSB induites par RAG et, par conséquent, une absence totale de lymphocyte mature (excepté dans le cas d'une déficience en XLF, voir ci-dessous), mais également (c) des anomalies du développement plus ou moins sévères et $(d)$ une prédisposition aux cancers [6]. Chez la souris, une déficience de la voie NHE) (par exemple une absence de XRCC4 ou LIG4) associée à un défaut du point de contrôle du 
cycle et de la mort cellulaire, dû à une absence du gène suppresseur de tumeur $p 53$, entraîne le développement rapide de lymphomes pro-B portant des translocations et des amplifications géniques impliquant l'oncogène $c$-myc et le locus codant la chaîne lourde des Ig. Les facteurs de la NHEJ sont donc à la fois essentiels à l'établissement du système immunitaire adaptatif et au maintien de l'intégrité du génome au cours de la recombinaison $\mathrm{V}(\mathrm{D}) \mathrm{J}$.

\section{XLF, un facteur à part dans la voie de réparation NHEJ}

XLF est un paralogue ${ }^{2}$ de XRCC4 avec lequel il partage une similarité structurale. II stimule le complexe XRCC4/LIG4 par un mécanisme encore incertain et, de manière indépendante, forme avec XRCC4 de longs filaments qui pourraient aider à la stabilisation des extrémités d'ADN, favorisant ainsi leur ligature par le complexe XRCC4/LIG4. Contrairement aux autres modèles murins déficients pour la voie NHEJ, les souris déficientes en XLF ont un système immunitaire relativement normal $[7,8]$. En effet, les lymphocytes déficients pour XLF sont capables de réparer les DSB induites par RAG, malgré une incapacité à réparer des DSB induites par des agents génotoxiques [8]. De plus, contrairement aux autres modèles déficients NHEJ/ p53, les animaux déficients XLF/p53 développent majoritairement des lymphomes T présentant peu d'instabilité génétique comme cela est le cas dans les lymphomes issus des animaux ayant une simple déficience en p53 [8]. Ces résultats montrent que XLF n'est pas nécessaire à la recombinaison $V(D)$ J et suggèrent qu'un autre facteur ou complexe permet de compenser son absence lors de la réparation des DSB induites par RAG [8]. L'un de ces complexes est la voie DDR dépendante d'ATM [9]. En effet, une déficience combinée de XLF

\footnotetext{
2 Deux gènes paralogues dérivent, suite à des évènements de duplication ou translocation, de gènes homologues issus d'un même gène ancestral.
}

et ATM conduit à un blocage du développement lymphocytaire dû à un défaut de réparation des DSB induites par $R A G$, démontrant l'existence d'une redondance fonctionnelle entre XLF et les facteurs de la DDR lors de la recombinaison $\mathrm{V}(\mathrm{D}) \mathrm{J}[\mathrm{9}]$.

\section{RAG joue un double rôle : couplage} entre clivage et réparation de l'ADN Plusieurs travaux antérieurs suggèrent que RAG joue un rôle après clivage de I'ADN [10]. Tout d'abord, les protéines RAGl/2 restent associées aux extrémités d'ADN dans le PCC. De plus, il a été montré que la partie C-terminale de RAG2 (acides aminés 352 à 527 chez la souris), qui n'est pas nécessaire pour la recombinaison $V(D)$ J, est cependant importante pour l'efficacité et la fidélité de la réaction de recombinaison. En effet, l'expression d'une forme tronquée de RAG2 (appelée coreRAG2) déstabilise le PCC in vitro et augmente la formation de produits de recombinaison aberrants in vivo. De plus, les souris exprimant coreRAG2, et conjointement déficientes en p53, développent des lymphomes thymiques agressifs présentant de nombreuses translocations chromosomiques impliquant les gènes codant le TCR et les Ig [11]. Ces tumeurs sont similaires à celles qui se développent chez les souris déficientes pour ATM [11]. Ces résultats suggèrent qu'à la fois ATM et le complexe RAG participent à la stabilisation des DSB. Nous avons alors émis l'hypothèse que les protéines RAG puissent aussi compenser l'absence de XLF au cours de la recombinaison $V(D)$ J. Pour tester cette hypothèse, nous avons généré des modèles murins exprimant coreRAG2 et déficientes en XLF [12]. De manière flagrante, les animaux déficients coreRAG2/XLF, contrairement aux simples mutants, présentent une immunodéficience sévère associée à un blocage du développement lymphocytaire à un stade précoce. Ce blocage est dû à une incapacité à réparer les DSB générées au cours de la recombinaison $V(D)$ J. De plus, les cassures non répa- rées, en l'absence de p53, entraînent une instabilité génomique importante et la formation de translocations chromosomiques. Nous avons également montré que les souris triple mutantes coreRAG2/ XLF/p53 développent des lymphomes pro-B portant des aberrations génomiques complexes impliquant les sites de recombinaison $V(D)$ J et l'oncogène c-myc [12]. Le complexe RAGl/2 et XLF sont donc fonctionnellement redondants lors de l'étape de réparation des DSB générées au cours de la recombinaison $V(D) J$. Nous suggérons que le complexe RAG, via la stabilisation des DSB au sein du PCC, et XLF, via la formation de filaments XLF/XRCC4, assurent tous deux le maintien des extrémités d'ADN après clivage et donc leur réparation par la voie NHEJ (Figure 1). Dans ce modèle, ATM contribue également à la stabilisation des DSB au sein du PCC [3]. Ceci est en accord avec nos résultats montrant que les lymphocytes déficients pour ATM et exprimant coreRAG2 n'ont pas de défaut accru de la recombinaison $V(D) J$ [12] dans ce cas, le filament XLF/XRCC4 est suffisant pour assurer le maintien des DSB (Figure 1).

Il a été depuis longtemps suggéré qu'un élément transposable - qui aurait intégré le génome d'un ancêtre commun à tous les vertébrés - pourrait être à l'origine de la recombinase $\mathrm{RAGl} / 2$ et donc du système immunitaire adaptatif. Cette hypothèse est d'ailleurs soutenue par la récente découverte chez l'Amphioxus du transposon protoRAG [13]. Ce transposon contient deux gènes très similaires à RAG1 et RAG2. De manière très surprenante, protoRAG2 ne contient pas la partie C-terminale de RAG2. Cette région contient plusieurs motifs dont un homéodomaine (PHD) capable de reconnaître la forme triméthylée de I'histone H3K4, une région acide importante pour guider les DSB vers la voie NHEJ et un signal de dégradation dépendant du cycle cellulaire. II est donc possible que l'acquisition de cette région régulatrice ait contribué à l'évolution d'une transposase vers une recombinase 
permettant la diversification du génome au profit du système immunitaire tout en préservant son intégrité [10].

\section{PAXX rejoint la partie}

Nous avons récemment découvert un autre exemple de redondance fonctionnelle au cours de la recombinaison $V(D)$ J [14]. PAXX est un paralogue de XRCC4 et XLF [15]. Les trois protéines ont de fortes similarités structurales, cependant le gène Paxx semble être apparu plus tardivement au cours de l'évolution, probablement via un événement de duplication génique. PAXX se localise sur les DSB et fonctionne avec XRCC4 et XLF pour assurer leur réparation et la survie cellulaire en réponse à des agents génotoxiques induisant des DSB. PAXX lui-même ne se lie pas à I'ADN, mais interagit avec Ku pour promouvoir l'assemblage des autres facteurs de la NHEJ et faciliter la ligature des extrémités d'ADN.

Pour tester le rôle potentiel de PAXX au cours de la recombinaison $V(D) J$, nous avons développé une méthodologie utilisant

la technique

CRISPR - Cas 9

$(\rightarrow)$ Voir la Synthèse de J.P. Tremblay, $m / s n^{\circ} 11$, novembre 2015, page 1014

[16] $(\rightarrow)$ per-

mettant de supprimer Paxx dans des lignées lymphocytaires $B$. Ce modèle cellulaire permet d'étudier la recombinaison $V(D)$ J dans des conditions pseudo-physiologiques. Nous avons ainsi pu montrer que PAXX n'est pas nécessaire pour la réparation des DSB induites par RAG [14]. En accord avec ces résultats, les souris déficientes pour PAXX ont un système immunitaire normal [17]. Cependant, une double déficience de PAXX et XLF abolit la réparation des $D S B$ générées par $R A G$, conduisant à un défaut sévère de la recombinaison $V(D) J[14]$. En absence de XLF, la fonction de PAXX ne se limite pas à la recombinaison $V(D) J$. En effet, chez la souris, une déficience combinée $P A X X / X L F$ conduit à une létalité embryonnaire associée à une apoptose massive des cellules du système ner- veux central et un blocage complet du développement lymphocytaire [17]. De plus, les cellules déficientes PAXX/XLF sont extrêmement sensibles au stress génotoxique induisant des DSB [14]. PAXX et XLF partagent donc une fonction qui est essentielle à la réparation des DSB et ceci dans plusieurs types cellulaires. De manière informative, PAXX n'est pas redondant avec ATM ou la partie C-terminale de RAG2. En effet, les cellules B déficientes pour PAXX/ ATM ou PAXX/coreRAG2 sont toujours capables de réaliser une recombinaison $V(D) J[14]$. Ces résultats divergent de ceux obtenus dans le contexte d'une déficience XLF/ATM ou XLF/coreRAG2 et démontrent que certaines des fonctions de PAXX et de son paralogue XLF sont différentes. Nous suggérons un modèle dans lequel XLF participe à la réparation des DSB via deux mécanismes (Figure 1). Un premier permet la stabilisation des extrémités d'ADN via la formation des filaments XLF-XRCC4; cette fonction est redondante avec celle du complexe RAG et ATM dans le cadre de la recombinaison $V(D) J$. Un deuxième mécanisme favorise la ligature des DSB par le complexe XRCC4LIG4 ; nous suggérons que PAXX et XLF sont redondants lors de cette étape (Figure 1). Cette redondance fonctionnelle explique certainement pourquoi des mutations génétiques du gène $P A X X$ n'ont pas encore été découvertes chez l'humain, notamment dans le cadre de pathologies sévères.

Dans l'ensemble, nos travaux permettent de mieux comprendre les mécanismes et processus évolutifs qui ont permis l'émergence du système immunitaire adaptatif chez les vertébrés. De plus, ils déterminent les multiples voies, souvent redondantes, qui permettent de diversifier le génome des cellules lymphoïdes tout en minimisant les risques d'instabilité génomique et de transformation néoplasique. $\diamond$

Paralogy and redundancy: maintaining genome integrity during $V(D) J$ recombination

\section{LIENS D'INTÉRÊT}

Les auteurs déclarent n'avoir aucun lien d'intérêt concernant les données publiées dans cet article.

\section{RÉFÉRENCES}

1. Hassanin A. Évolution des séquences «signal de recombinaison » dans le locus de la région variable de la chaîne lourde des immunoglobulines. Med Sci (Paris) $2001 ; 17: 1168-75$.

2. Schatz DG, Swanson PC. V(D)J recombination: mechanisms of initiation. Annu Rev Genet 2011 ; 45 : 167-202.

3. Helmink BA, Sleckman BP. The response to and repair of RAG-mediated DNA double-strand breaks. Annu Rev Immunol 2012 ; 30 : 175-202.

4. Deriano L, Roth DB. Modernizing the nonhomologous end-joining repertoire: alternative and classical NHE, share the stage. Annu Rev Genet 2013 ; 47 : 433-55.

5. Revy P, de Villartay JP. Cernunnos, un nouveau facteur de la réparation de l'ADN essentiel pour le système immunitaire. Med Sci (Paris) 2006 ; 22 : 569-70.

6. Revy P, Buck D, le Deist F, de Villartay JP. The repair of DNA damages/modifications during the maturation of the immune system: lessons from human primary immunodeficiency disorders and animal models. $A d v$ Immunol $2005 ; 87$ : 237-95.

7. Vera G, Rivera-Munoz P, Abramowski V, et al. Cernunnos deficiency reduces thymocyte lifespan and alters the T cell repertoire in mice and humans. Mol Cell Biol 2012 ; 33 : 701-11.

8. Li G, Alt FW, Cheng HL, et al. Lymphocyte-specific compensation for XLF/cernunnos end-joining functions in V(D)J recombination. Mol Cell 2008 ; 31 : 631-40.

9. Kumar V, Alt FW, Oksenych V. Functional overlaps between XLF and the ATM-dependent DNA double strand break response. DNA Repair 2014 ; 16 : 11-22.

10. Lescale C, Deriano L. The RAG recombinase: Beyond breaking. Mech Ageing Dev 2016 ; doi: 10.1016/j. mad.2016.11.003.

11. Deriano L, Chaumeil J, Coussens M, et al. The RAG2 C-terminus suppresses genomic instablity and lymphomagenesis. Nature 2011 ; 471 : 119-23.

12. Lescale C, Abramowski V, Bedora-Faure M, et al. RAG2 and XLF/Cernunnos interplay reveals a novel role for the RAG complex in DNA repair. Nat Commun 2016 ; 7 10529.

13. Huang S, Tao X, Yuan S, et al. Discovery of an active RAG transposon illuminates the origins of $V(D) J$ recombination. Cell 2016; 166 : 102-14.

14. Lescale C, Lenden Hasse H, Blackford AN, et al. Specific roles of XRCC4 paralogs PAXX and XLF during V(D)J recombination. Cell Rep 2016 ; 16 : 2967-79.

15. Ochi T, Blackford AN, Coates J, et al. DNA repair. PAXX, a paralog of XRCC4 and XLF, interacts with Ku to promote DNA double-strand break repair. Science $2015 ; 347: 185-8$.

16. Tremblay JP. CRISPR, un système qui permet de corriger ou de modifier l'expression de gènes responsables de maladies héréditaires. Med Sci (Paris) 2015 ; 31 : 1014-22.

17. Balmus G, Barros AC, Wijnhoven PW, et al. Synthetic lethality between PAXX and XLF in mammalian development. Genes Dev 2016 ; 30 : 2152-7. 